\title{
Feedback control of polyketide metabolism during tylosin production
}

\author{
Andrew R. Butler, $†$ Simon A. Flint $†$ and Eric Cundliffe \\ Author for correspondence: Eric Cundliffe. Tel: +44 116252 3451. Fax: +44 1162523369. \\ e-mail: ec13@le.ac.uk
}

Department of Biochemistry, University of Leicester, Leicester LE1 7RH, UK
Tylosin is produced by Streptomyces fradiae via a combination of polyketide metabolism and synthesis of three deoxyhexose sugars, of which mycaminose is the first to be added to the polyketide aglycone, tylactone (protylonolide). Previously, disruption of the gene (tyIMII) encoding attachment of mycaminose to the aglycone unexpectedly abolished accumulation of the latter, raising the possibility of a link between polyketide metabolism and deoxyhexose biosynthesis in S. fradiae. However, at that time, it was not possible to eliminate an alternative explanation, namely, that downstream effects on the expression of other genes, not involved in mycaminose metabolism, might have contributed to this phenomenon. Here, it is shown that disruption of any of the four genes (tyIMI-III and tylB) specifically involved in mycaminose biosynthesis elicits a similar response, confirming that production of mycaminosyl-tylactone directly influences polyketide metabolism in S. fradiae. Under similar conditions, when mycaminose biosynthesis was specifically blocked by gene disruption, accumulation of tylactone could be restored by exogenous addition of glycosylated tylosin precursors. Moreover, certain other macrolides, not of the tylosin pathway, were also found to elicit qualitatively similar effects. Comparison of the structures of stimulatory macrolides will facilitate studies of the stimulatory mechanism.

Keywords: mycaminose biosynthesis, polyketide, Streptomyces fradiae, tylactone, tylosin production

\section{INTRODUCTION}

Tylosin, a macrolide antibiotic produced by Streptomyces fradiae, consists of a polyketide lactone substituted with three 6-deoxyhexose sugars. The TylG polyketide synthase (PKS) produces and cyclizes the aglycone (tylactone, also known as protylonolide), which is subsequently oxidized at C-20 and C-23 to generate tylonolide and concurrently substituted with three deoxyhexose sugars (D-mycaminose, 6-deoxy-Dallose and L-mycarose; Fig. 1). These are added in a preferred but not obligatory order, although (importantly, in the present context) mycaminose always goes on first. Finally, the deoxyallose moiety is converted to D-mycinose via stepwise bis-O-methylation, thereby generating tylosin (for a review, see Baltz \& Seno, 1988).

† These authors made equal contributions to this work.

Abbreviations: OMT, O-mycaminosyltylonolide; PKS, polyketide synthase.
Elucidation of the biochemical route to tylosin relied heavily on blocked $S$. fradiae mutants, which displayed nine distinct phenotypes in cross-feeding analysis and eventually allowed 13 genetic loci $(t y l A-M)$ to be mapped by complementation with cloned fragments of tyl DNA (Fishman et al., 1987; Beckmann et al., 1989).

More recently, the entire $t y l$ gene cluster $(\sim 85 \mathrm{~kb})$ has been sequenced (Szoke et al., 1989; Rosteck et al., 1991; Merson-Davies \& Cundliffe, 1994; Gandecha \& Cundliffe, 1996; Gandecha et al., 1997; Wilson \& Cundliffe, 1998; Bate \& Cundliffe, 1999; Bate et al., 1999, 2000; Fouces et al., 1999; Wilson \& Cundliffe, 1999; see also GenBank accession no. U78289), revealing five mega genes (total $\sim 41 \mathrm{~kb}$ ) that encode the TylG PKS, flanked by sugar biosynthetic genes, ancillary genes, regulatory elements and resistance determinants (for a review, see Cundliffe, 1999). The present work is focused on the four genes involved in mycaminose biosynthesis, three of which (tylMI-III) are located immediately downstream of the $t y l G$ group, about $45 \mathrm{~kb}$ 
Tylactone (Protylonolide)<smiles>CCC(OC(=O)CC(C)C(C)C(O)CC(C)C(=O)/C=C/C(C)C)C(C)C</smiles>

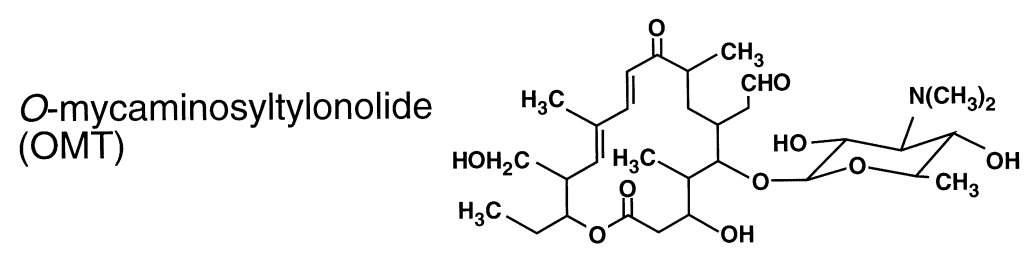

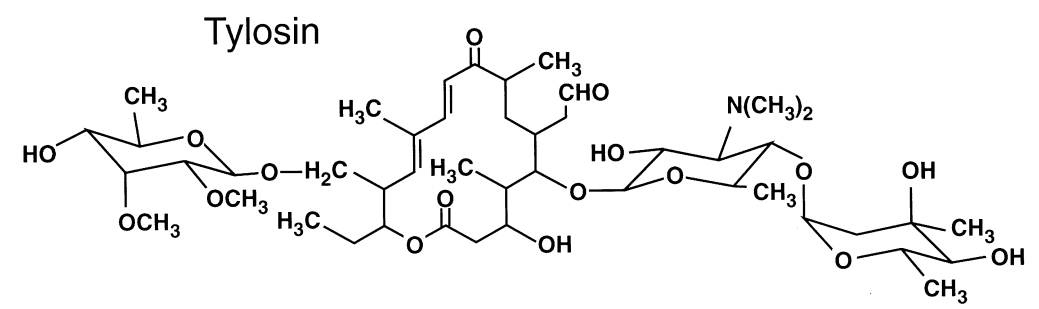

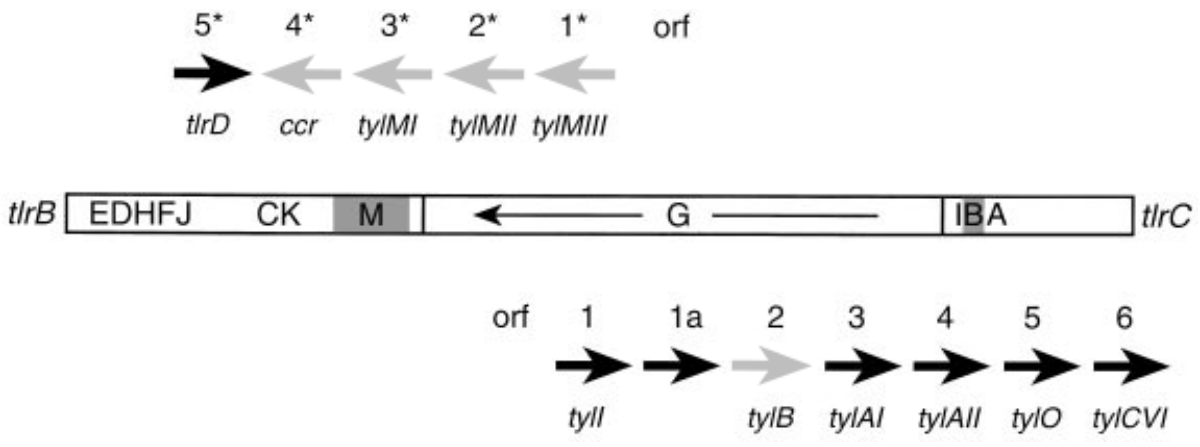

Fig. 2. Tylosin-biosynthetic gene cluster. Not drawn to scale. The bar represents the entire tyl cluster ( $\sim 85 \mathrm{~kb})$ showing the various tyl loci flanked by the resistance genes $\operatorname{lr} B$ and $\operatorname{t} / \mathrm{rC}$. The tylG locus represents five mega genes ( $\sim 41 \mathrm{~kb})$ encoding the PKS complex. Upstream of tylG lie 12 ORFs (including $t / r C$ ), seven of which are shown here. Downstream of tyIG lie 26 ORFs including $t / r B$, five of which are shown here. These are designated $1 *$, $2^{*}$, etc. to distinguish them from ORFs on the other side of tylG. Genes analysed here are shown as grey arrows and their locations within the cluster are indicated on the bar.

distant from their functional partner $(t y l B)$, which curiously lies on the other side of $t y l G$ (Fig. 2). Although the tylL locus was originally mapped to the region occupied by tylMI-III (cited in Fishman et al., 1987) and although a tylL mutant was found to harbour an opal mutation in tylMII (Clark, 1997), the TylL phenotype (i.e. failure to synthesize or add any of the three tylosin sugars) cannot readily be explained by impairment of the function of any one of the $t y l M$ genes and, therefore, probably results from multiple mutations. For that reason, the ' $t y l L$ ' locus is not represented in Fig. 2.

The present work arises from earlier studies (Fish \& Cundliffe, 1997) in which tylMII (inappropriately referred to as 'tylM2' at that time) was disrupted in the genome of $S$. fradiae. This gene (orf $2 *$ in the systematic nomenclature, see Fig. 2) encodes mycaminosyltransferase (Gandecha et al., 1997), the enzyme that normally adds the first sugar during tylosin production. It was therefore expected that the tylMII-disrupted strain, SF01, would be unable to produce tylosin or any of its glycosylated precursors, but would still retain the ability to bioconvert such precursors to tylosin. Such proved to be the case but, surprisingly, strain SF01 did not accumulate tylactone, except when fed exogenously with glycosylated macrolides such as O-mycaminosyltylonolide (OMT). In contrast, when genes involved in the biosynthesis or addition of mycinose or mycarose were disrupted (Wilson \& Cundliffe, 1998; Bate et al., 2000), the predicted products (demycinosyl-tylosin and demycarosyl-tylosin, respectively) accumulated. Although no unequivocal explanation was offered for 
the results obtained with strain SF01, it seemed possible that tylactone production was somehow stimulated (mechanism unspecified) by glycosylated macrolides. As an added complication, however, impairment of polyketide metabolism in strain SF01 in the absence of glycosylated compounds might not have resulted exclusively from disruption of tylMII per se, but might have involved possible downstream effects - particularly on expression of orf $4^{*}(\mathrm{ccr})$. This gene encodes crotonyl-CoA reductase with a likely role in production of butyryl-CoA, the 4-carbon extender substrate that provides carbons 5, 6, 19 and 20 of tylactone (Gandecha et al., 1997). The aim of the present work was to resolve this matter and to examine further the apparent influence of glycosylated macrolides on polyketide metabolism in $S$. fradiae.

\section{METHODS}

Bacterial strains and genetic manipulations. S. fradiae T59235 (also known as C373.1; referred to here as wild-type) and derivatives thereof were propagated at $30^{\circ} \mathrm{C}$ in tryptic soy broth (TSB; Difco) in sprung flasks or at $37^{\circ} \mathrm{C}$ on AS- 1 agar plates (Wilson \& Cundliffe, 1998) and maintained at $-70{ }^{\circ} \mathrm{C}$ as mycelial fragments following addition of DMSO $(5 \%, \mathrm{v} / \mathrm{v}$; final concentration) to TSB-grown cultures. Plasmids were manipulated in Escherichia coli $\mathrm{DH} 5 \alpha$ using standard protocols (Sambrook et al., 1989). DNA was introduced into $S$. fradiae by conjugal transfer from E. coli S17-1 as described elsewhere (Fish \& Cundliffe, 1997) using pOJ260 (Bierman et al., 1992) and pLST9828 (Butler et al., 1999). pOJ260 is incapable of replication in Streptomyces spp. and was used for targeted gene disruption. pLST9828, used for complementation analysis, integrates with high efficiency into the chromosomal $\Phi \mathrm{C} 31 \mathrm{attB}$ site and contains a powerful constitutive promoter, ermEp* (Bibb et al., 1994), for expression of inserted DNA. Both vectors carry apramycin-resistance markers.

Targeted gene disruption via gene transplacement. Fragments of $t y l$ DNA, each containing a specific mycaminosebiosynthetic gene, were ligated into pIJ2925 (Janssen \& Bibb, 1993). Each of these DNA fragments contained a central, unique restriction site located within the cloned gene, into which the hygromycin B resistance cassette, $\Omega$ hyg $(2 \cdot 3 \mathrm{~kb}$, with flanking transcriptional terminators; Blondelet-Rouault et al., 1997), was inserted via blunt-end ligation. The complete insert was then excised using BglII sites which flank the pIJ2925 polylinker and ligated into the BamHI site of pOJ 260. Following conjugal transfer into $S$. fradiae, transconjugants were selected on hygromycin B and then screened for apramycin sensitivity (Fish \& Cundliffe, 1997) to identify those in which chromosomal target genes had been replaced with disrupted constructs via double recombination. The gene disruption constructs were assembled as follows.

Disruption of tylMIII (orf1*). A 2126 bp AgeI fragment from cosmid pMOMT4 (Beckmann et al., 1989) was cloned in $\mathrm{pIJ} 2925$. Insertion of $\Omega$ hyg at the unique $M l u \mathrm{I}$ site interrupted the sequence encoding TylMIII (normally 423 amino acids) $291 \mathrm{bp}$ downstream of the proposed translational start.

Disruption of tylMII (orf2*). A strain disrupted in tylMII and designated SF01 was already available for analysis (Fish \& Cundliffe, 1997).

Disruption of tylMI (orf3*). A 1546 bp HincII fragment from pMOMT4 was ligated into pIJ2925 and the unique MscI site provided a disruption site for tylMI, 256 bp downstream of the proposed translational start. The deduced length of intact TylMI is 254 amino acid residues.

Disruption of ccr (orf4*). A 1911 bp StyI fragment from pMOMT4 was ligated into pIJ2925 and $\Omega$ hyg was inserted into the BsaAI site. This interrupted the $c c r$ coding sequence $168 \mathrm{bp}$ downstream of the translational start. The deduced length of intact Ccr is 449 amino acid residues.

Disruption of tylB (orf2). A $2251 \mathrm{bp} \mathrm{HincII}$ fragment from pSET552 (Beckmann et al., 1989) was ligated into pIJ2925 and Shyg was inserted at a unique BstEII site, thereby disrupting the $t y l B$ coding sequence 638 bp downstream from its putative translational start. The truncated gene product was predicted to be 212 amino acids in length compared with 388 amino acid residues for the intact protein.

Authentication of disrupted strains. Southern blot hybridization analysis, using the Boehringer Mannheim DIG High Prime DNA Labelling and Detection Starter Kit II, was used to confirm each of the gene disruptions using probes specific to the respective target genes.

Complementation of disrupted strains. To compensate for possible effects on the expression of downstream genes in the tylB-disrupted strain, a 3118 bp $M l u \mathrm{I}-E c o \mathrm{RV}$ fragment of $t y l$ DNA from pSET552 (together with ermEp*) was inserted into the chromosomal ФC31 attB site using pLST9828. The complementing DNA fragment contained the co-directional genes tylAI, tylAII and tylO together with flanking sequences (139 bp upstream of $t y l A I$ and $144 \mathrm{bp}$ downstream of $t y l O)$ and was oriented favourably for control by ermEp*.

Tylosin production fermentation and metabolite analysis. Fermentation and HPLC analysis of the products was carried out as described elsewhere (Butler et al., 1999) except that, for convenience, $0.5 \%(\mathrm{w} / \mathrm{v})$ corn steep solids (Sigma) replaced $1.0 \%(\mathrm{v} / \mathrm{v})$ corn steep liquor in pre-fermentation media used for some later fermentations. Control experiments revealed no detectable impact of this change on extracted fermentation products. In bioconversion studies, tylosin precursors or other macrolides (10 mg each, dissolved in $100 \mu \mathrm{l}$ DMSO) were added to fermentations (50 ml cultures) after $2 \mathrm{~d}$ and incubation was continued for a further $5 \mathrm{~d}$ before analysis. DMSO was added to control fermentations.

\section{RESULTS AND DISCUSSION}

\section{Disruption of ccr (orf4*)}

This was achieved via targeted gene replacement (involving double recombination) using the hygromycin B resistance cassette, $\Omega$ hyg (Blondelet-Rouault et al., 1997). Having confirmed the disruption ('knockout') by Southern analysis, the resultant ' $c c r-K O$ ' strain was fermented and the products were examined by HPLC (Fig. 3). Since the disrupted strain still produced significant, although reduced, amounts of tylosin, the ccr gene product was evidently not essential for tylosin production under these conditions. Interestingly, the $c c r$-specific probe that was used to confirm the authenticity of the knockout strain also found a second hybridization target at high stringency (presumably, another $c c r$ gene) elsewhere in the $S$. fradiae genome. We do not know whether any requirement for Ccr activity during tylosin production under these conditions can be satisfied (partially or otherwise) by the product of the 


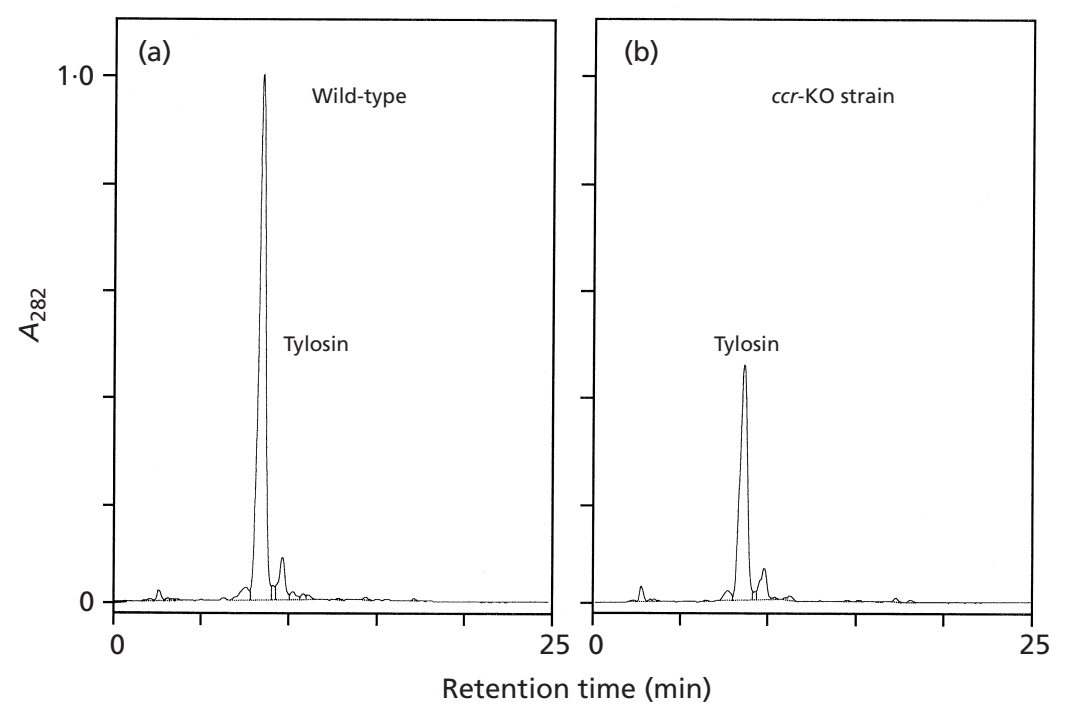

Fig. 3. Fermentation products from $S$. fradiae wild-type and the ccr-disrupted strain. HPLC analysis of material produced by (a) wild-type and (b) ccr (orf4*)-disrupted strain.

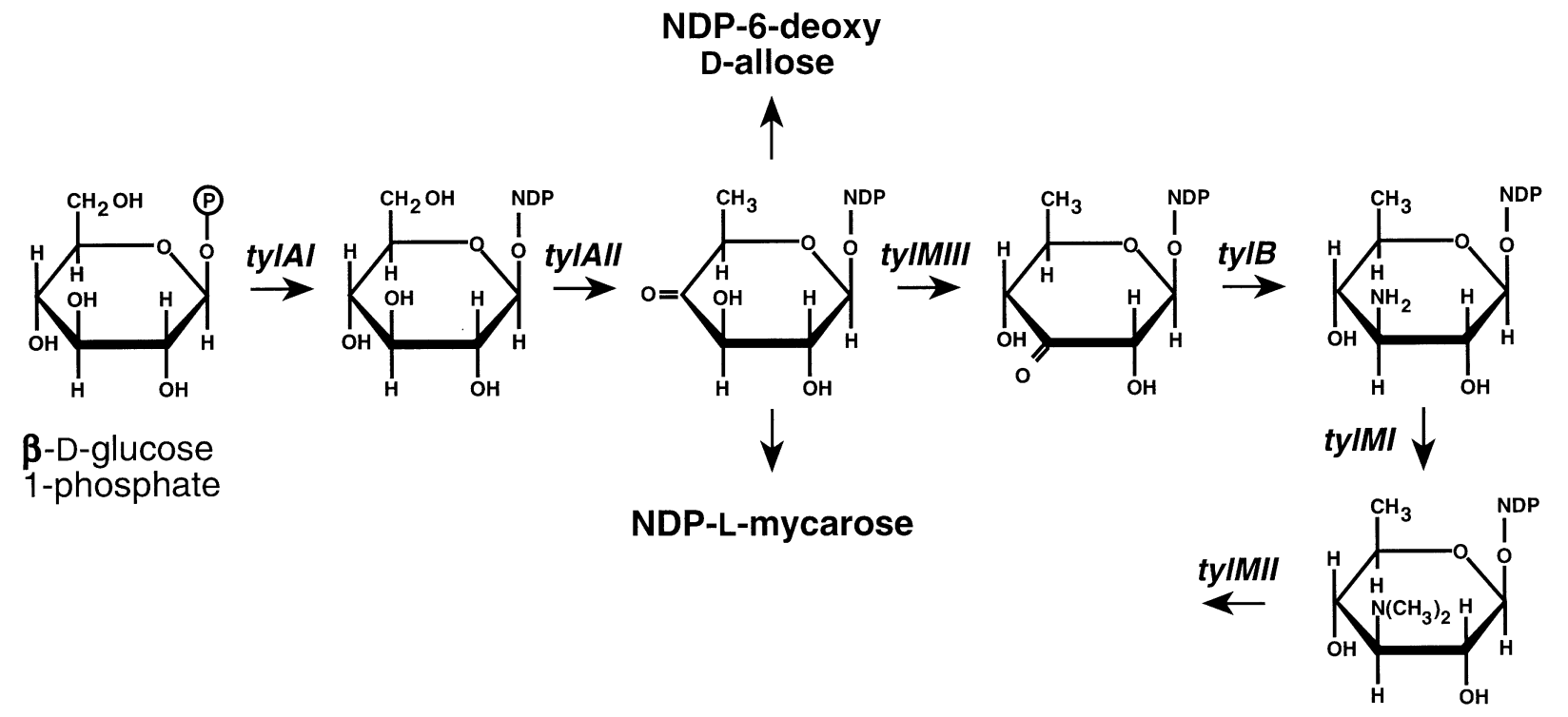

NDP-D-mycaminose

Fig. 4. Biosynthetic route to mycaminose. The pathway represented here begins with the two reactions common to the synthesis of all three sugar moieties of tylosin.

'other' gene but, in any event, impaired expression of orf $4 *$ could not have accounted for the failure of strain SF01 to accumulate tylactone in earlier studies.

\section{Disruption of mycaminose-biosynthetic genes}

Targeted gene replacement, again using $\Omega$ hyg, was employed to disrupt (separately) tylMI, tylMIII and $t y l B$ in the genome of $S$. fradiae and the respective disruptions were confirmed by hybridization analysis using probes specific to each of the target genes (data not shown). The latter have been proposed (see Cundliffe, 1999) to encode methyltransferase (product of tylMI), putative 3,4- isomerase (product of tylMIII) and aminotransferase (TylB) activities, which, together with mycaminosyltransferase (encoded by tylMII), constitute the entire complement of enzymes specifically required for the biosynthesis and addition of mycaminose during tylosin production (Fig. 4). When fermentation products from the three disrupted strains (designated tylMI-KO, $t y l M I I I-\mathrm{KO}$ and $t y l B-\mathrm{KO}$ ) were analysed by HPLC, the results were similar and resembled those previously observed with strain SF01 (tylMII-KO). None of these strains produced tylosin (confirming for the first time that orf 1 " is indeed a ' $t y l$ ' gene), nor did they accumulate tylactone unless a glycosylated macrolide (here, OMT) 

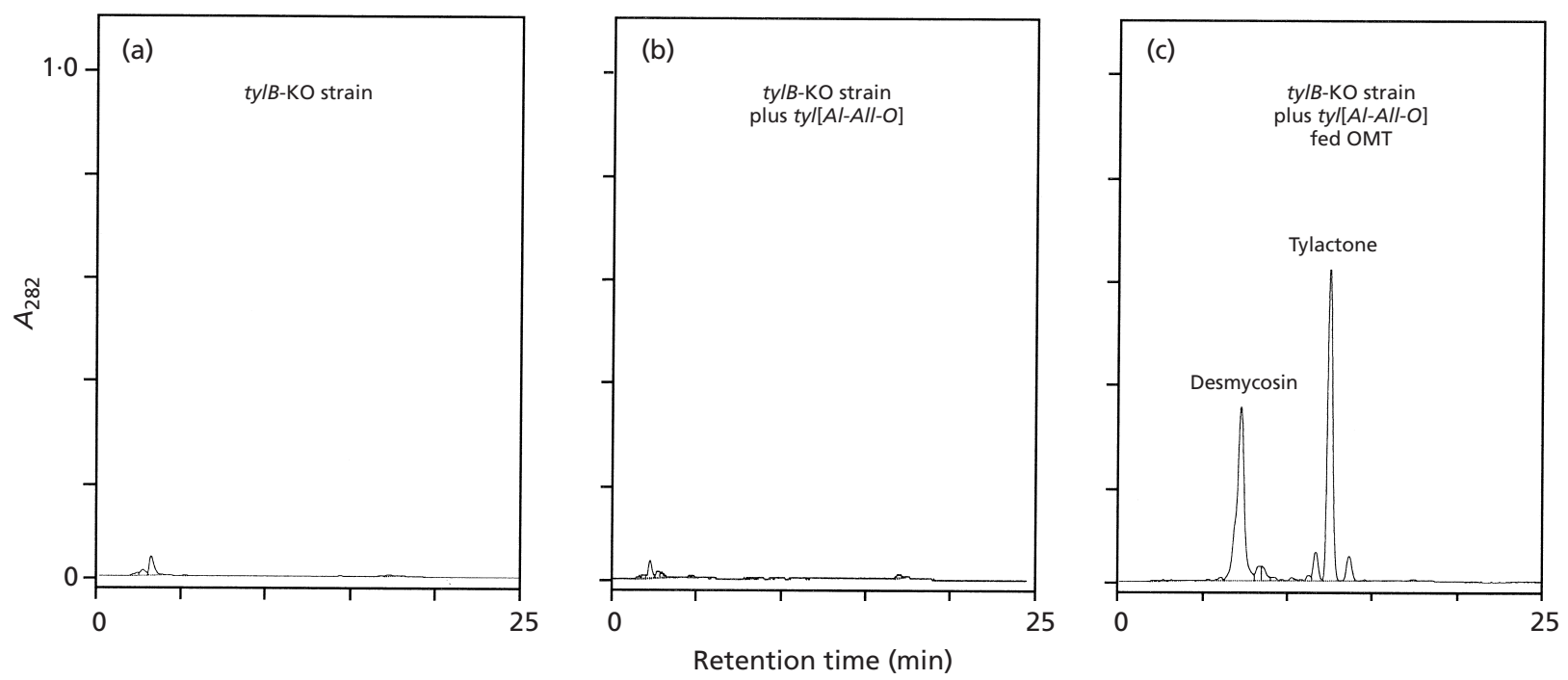

Fig. 5. OMT-induced tylactone biosynthesis in the tylB-disrupted strain. HPLC analysis of material produced during fermentation of: (a) tylB-disrupted strain; (b) tylB-disrupted strain complemented with tylAI+tylAII+tylO; (c) tylBdisrupted strain complemented with tylAI+tylAII+tylO and fed $10 \mathrm{mg}$ OMT.

was added exogenously to the fermentation medium. Only data obtained with the $t y l B-K O$ strain are shown here (Fig. 5). Again, it was necessary to control for possible downstream effects resulting from disruption of the target gene. Thus, tylAI and tylAII (inappropriately designated $t y l A 1$ and $t y l A 2$ earlier; Merson-Davies \& Cundliffe, 1994) lie immediately downstream of $t y l B$ (Fig. 2) and encode enzymes common to the biosynthesis of all three tylosin sugars (Fig. 4). Also, and more importantly in the present context, tylO encodes an 'editing' thioesterase that greatly affects the level of tylactone production (Butler et al., 1999). However, when a DNA fragment containing [tylAI-tylAII-tylO] under control of the strong constitutive promoter ermEp* was integrated into the genome of the $t y l B-\mathrm{KO}$ strain, the resultant organism remained unable to accumulate tylactone except in the presence of exogenous OMT (Fig. 5b, c). Under the latter conditions, the added OMT was converted to desmycosin (demycarosyl-tylosin) and not to tylosin. This was not unexpected since orf6 (downstream of, and co-directional with, $t y l B)$ had previously been characterized as a mycarose-biosynthetic gene, i.e. tylCVI (Bate et al., 2000). Evidently, orf2 (tylB) and orf6 (tylCVI) are normally co-transcribed.

Two conclusions follow from these data. Firstly, failure of these various disrupted strains to accumulate tylactone was a direct consequence of the impairment of mycaminose biosynthesis and did not result indirectly from effects on the expression of genes downstream of the respective knockout targets. Secondly, tylosin precursors such as OMT (and, to a lesser extent, tylosin itself; see Fish \& Cundliffe, 1997) exert a positive influence on tylactone production in the various knockout strains. Presumably, in wild-type $S$. fradiae, tylactone biosynthesis proceeds at only minimal levels unless the product gets glycosylated, in which case a trickle becomes a flood.

\section{Effects of macrolides other than tylosin precursors}

It was argued previously (Fish \& Cundliffe, 1997) that accumulation of tylactone in strain SF01 after feeding with OMT could not be due to degradation of OMT, since cleavage of the latter would release tylonolide not tylactone. This point was reinforced in the present work when tylactone accumulation in the tylMIIdisrupted strain, SF01, was triggered by OMT at inputs too low to be detected by HPLC following re-extraction from fermentation cultures (data not shown). It was also observed that accumulation of tylactone in this strain could be provoked, albeit with differing efficiencies, by glycosylated macrolides other than tylosin precursors, including rosaramicin and spiramycin, but not by chalcomycin, erythromycin or carbomycin (Fig. 6). Although these data do not reveal the structural features needed to elicit this effect, it is clear that the accumulated tylactone could not have been derived from the added compounds.

\section{Summary and conclusions}

The present data establish that tylactone biosynthesis in $S$. fradiae is controlled by glycosylated macrolides, although it is not clear whether stimulation of polyketide metabolism by OMT, etc. involves activation or derepression of $t y l G$ expression. In other work, tylactone production was shown to require expression of the regulatory gene $t y l R$, disruption of which prevented $S$. fradiae from accumulating tylactone even in the presence 

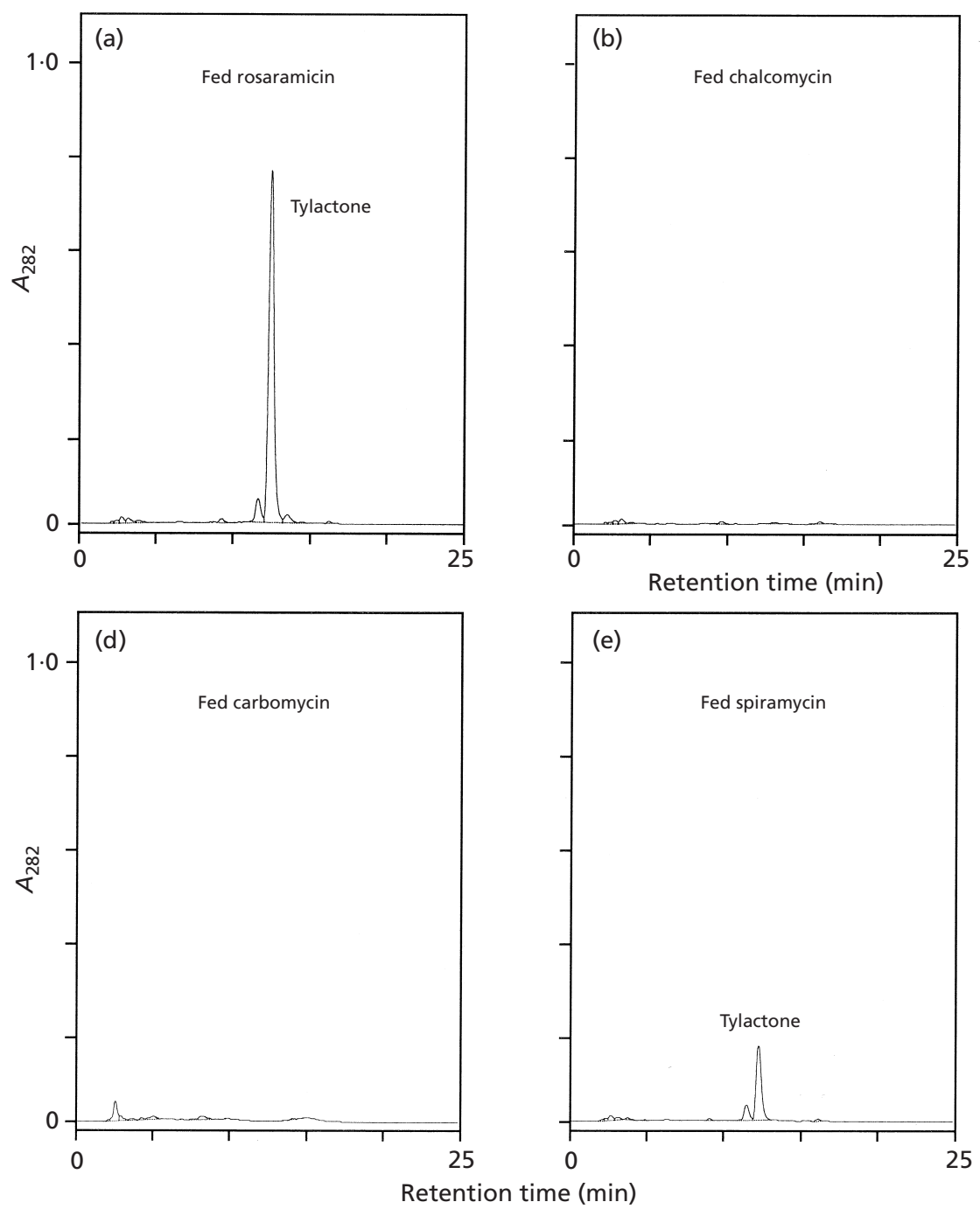
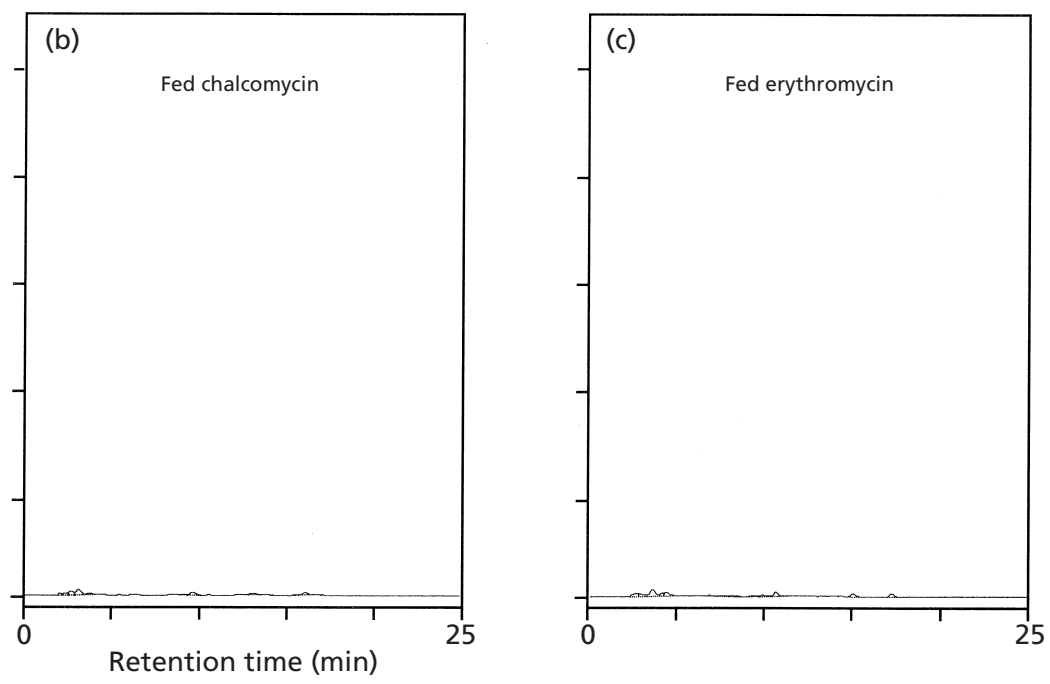

Fig. 6. Stimulation of tylactone synthesis in the tylMII-disrupted strain (SF01) by exogenous glycosylated macrolides other than tylosin precursors. HPLC analysis of material produced during fermentation of the ty/MII-disrupted strain fed with $10 \mathrm{mg}$ of: (a) rosaramicin; (b) chalcomycin; (c) erythromycin; (d) carbomycin; (e) spiramycin. of exogenously added OMT (Bate et al., 1999). Since $t y l R$ appears to encode a positive regulator, it is possible that efficient transcription of $t y l G$ requires the TylR protein together with glycosylated macrolides as cofactors. Moreover, expression of $t y l R$ is also needed for deoxyhexose metabolism (and, in particular, for mycaminose biosynthesis) in $S$. fradiae, since tylactone was not glycosylated when fed exogenously to a $t y l R$ disrupted strain (Bate et al., 1999). These data could be reconciled if TylR were to regulate the synthesis of mycaminose (and the other tylosin sugars?) in concert with tylactone production, in which case it would be instructive to know whether OMT also influences deoxyhexose metabolism in $S$. fradiae, perhaps by binding to TylR.

In conclusion, the manner in which glycosylated macrolides stimulate tylactone production in $S$. fradiae is not yet understood. Future studies will centre on the (presumed) DNA-binding properties of the TylR protein and on attempts to identify OMT-binding regulatory protein(s).

\section{ACKNOWLEDGEMENTS}

This work was supported by BBSRC (grant number 91/ T08195), by Lilly Research Laboratories, Indianapolis, USA, and by a BBSRC research studentship awarded to S.A.F.

\section{REFERENCES}

Baltz, R. H. \& Seno, E. T. (1988). Genetics of Streptomyces fradiae and tylosin biosynthesis. Annu Rev Microbiol 42, 547-574.

Bate, N. \& Cundliffe, E. (1999). The mycinose-biosynthetic genes of Streptomyces fradiae, producer of tylosin. J Ind Microbiol Biotechnol 23, 118-122.

Bate, N., Butler, A. R., Gandecha, A. R. \& Cundliffe, E. (1999). Multiple regulatory genes in the tylosin biosynthetic cluster of Streptomyces fradiae. Chem Biol 6, 617-624.

Bate, N., Butler, A. R., Smith, I. P. \& Cundliffe, E. (2000). The mycarose-biosynthetic genes of Streptomyces fradiae, producer of tylosin. Microbiology 146, 139-146.

Beckmann, R. J., Cox, K. \& Seno, E. T. (1989). A cluster of tylosin biosynthetic genes is interrupted by a structurally unstable segment containing four repeated sequences. In Genetics and 
Molecular Biology of Industrial Microorganisms, pp. 176-186. Edited by C. L. Hershberger, S. W. Queener \& P. L. Skatrud. Washington, DC: American Society for Microbiology.

Bibb, M. J., White, J., Ward, J. M. \& Janssen, G. R. (1994). The mRNA for the $23 \mathrm{~S}$ rRNA methylase encoded by the ermE gene of Saccharopolyspora erythraea is translated in the absence of a conventional ribosome-binding site. Mol Microbiol 14, 533-545.

Bierman, M., Logan, R., O’Brien, K., Seno, E. T., Rao, R. N. \& Schoner, B. E. (1992). Plasmid cloning vectors for the conjugal transfer of DNA from Escherichia coli to Streptomyces spp. Gene 116, 43-49.

Blondelet-Rouault, M.-H., Weiser, J., Lebrihi, A., Branny, P. \& Pernodet, J.-L. (1997). Antibiotic resistance cassettes derived from the $\Omega$ interposon for use in E. coli and Streptomyces. Gene 190, 315-317.

Butler, A. R., Bate, N. \& Cundliffe, E. (1999). Impact of thioesterase activity on tylosin biosynthesis in Streptomyces fradiae. Chem Biol 6, 287-292.

Clark, S. L. (1997). Analysis of the tylLM region of the Streptomyces fradiae chromosome. $\mathrm{PhD}$ thesis, University of Leicester.

Cundliffe, E. (1999). Organization and control of the tylosinbiosynthetic genes of Streptomyces fradiae. Actinomycetologica $13,68-75$.

Fish, S. A. \& Cundliffe, E. (1997). Stimulation of polyketide metabolism in Streptomyces fradiae by tylosin and its glycosylated precursors. Microbiology 143, 3871-3876.

Fishman, S. E., Cox, K., Larson, J. L., Reynolds, P. A., Seno, E. T., Yeh, W.-K., Van Frank, R. \& Hershberger, C. L. (1987). Cloning genes for the biosynthesis of a macrolide antibiotic. Proc Natl Acad Sci U S A 84, 8248-8252.

Fouces, R., Mellado, E., Díez, B. \& Barredo, J. L. (1999). The tylosin biosynthetic cluster from Streptomyces fradiae: genetic organization of the left region. Microbiology 145, 855-868.
Gandecha, A. R. \& Cundliffe, E. (1996). Molecular analysis of $t l r D$, an MLS resistance determinant from the tylosin producer, Streptomyces fradiae. Gene 180, 173-176.

Gandecha, A. R., Large, S. L. \& Cundliffe, E. (1997). Analysis of four tylosin biosynthetic genes from the tylLM region of the Streptomyces fradiae genome. Gene 184, 197-203.

Janssen, G. R. \& Bibb, M. J. (1993). Derivatives of pUC18 that have $B g l I I$ sites flanking a modified multiple cloning site and that retain the ability to identify recombinant clones by visual screening of Escherichia coli colonies. Gene 124, 133-134.

Merson-Davies, L. A. \& Cundliffe, E. (1994). Analysis of five tylosin biosynthetic genes from the tylIBA region of the Streptomyces fradiae genome. Mol Microbiol 13, 349-355.

Rosteck, P. R., Jr, Reynolds, P. A. \& Hershberger, C. L. (1991). Homology between proteins controlling Streptomyces fradiae tylosin resistance and ATP-binding transport. Gene 102, 27-32.

Sambrook, J., Fritsch, E. F. \& Maniatis, T. (1989). Molecular Cloning: a Laboratory Manual, 2nd edn. Cold Spring Harbor, NY : Cold Spring Harbor Laboratory.

Szoke, P. A., Reynolds, P. A., Rosteck, P. R., Jr, Kreuzman, A. J., Yeh, W.-K., Becker, G. W. \& Hershberger, C. L. (1989). Sequence analysis of tylosin biosynthetic genes. In Abstracts of Posters of the Annual Meeting of the American Society for Microbiology, New Orleans. Abstract number H10.

Wilson, V. T. W. \& Cundliffe, E. (1998). Characterization and targeted disruption of a glycosyltransferase gene in the tylosin producer, Streptomyces fradiae. Gene 214, 95-100.

Wilson, V. T. W. \& Cundliffe, E. (1999). Molecular analysis of $t r B$, an antibiotic-resistance gene from tylosin-producing Streptomyces fradiae, and discovery of a novel resistance mechanism. J Antibiot 52, 288-296.

Received 10 November 2000; accepted 5 January 2001. 